\title{
Recent results of the pion-nucleon scattering and the widths of the delta and Roper resonances in BChPT
}

\author{
J. Gegelia ${ }^{1,2, *}$ \\ ${ }^{1}$ Institute for Advanced Simulation, Institut für Kernphysik and Jülich Center for Hadron Physics, \\ Forschungszentrum Jülich, D-52425 Jülich, Germany \\ ${ }^{2}$ Tbilisi State University, 0186 Tbilisi, Georgia
}

\begin{abstract}
Recent calculations of the pion-nucleon scattering in covariant baryon chiral perturbation theory with explicit delta resonances are presented. Two-loop results of the widths of the $\Delta$ and Roper resonances are discussed in the framework of an effective field theory with nucleons, pions and the Roper and delta resonances as dynamical degrees of freedom.
\end{abstract}

\section{Introduction}

Usage of chiral effective field theory (EFT) is based on a very well motivated assumption that quantum chromodynamics (QCD) is a correct theory of the strong interaction. Perturbative QCD is not applicable at low energies. Chiral EFT provides with a solution to this problem. This approach started with Ref. [1]. Based on the symmetries of QCD, chiral EFT aims at reproducing the $S$-matrix of QCD in low-energy region. Effective degrees of freedom of chiral EFT are hadrons: the pions, nucleons, $\Delta(1232)$, etc. Chiral EFT provides with a systematic expansion of physical quantities in powers of (small scale(s))/(large scale(s)). Most general EFT Lagrangian of hadrons with symmetries of QCD gives the most general $S$-matrix with these symmetries. To obtain $S$-matrix of QCD one needs to fix properly the parameters of EFT - a finite number of them to achieve finite accuracy. The procedure to be followed in the one baryon sector of the chiral EFT can be briefly summarized as follows:

- Write down the most general effective Lagrangian.

- Consider all Feynman diagrams contributing to the process in question. There are an infinite number of diagrams contributing to any scattering process. We need some organizing rule (power counting) which tells us relative importance of each diagram.

- Renormalize/subtract loop diagrams.

- Apply power counting - assigning orders of small parameter(s) to renormalized diagrams.

- Sum up all renormalized diagrams contributing up to given order.

- Only a finite number of diagrams contribute at any finite order in one-baryon sector.

Below we present recent results of two-loop calculations of the widths of the $\Delta$ and Roper resonances and a one-loop calculation of the pion-nucleon scattering in covariant baryon chiral perturbation theory (BChPT) with explicit delta resonances. Effective Lagrangian of

\footnotetext{
*e-mail: j.gegelia@fz-juelich.de
} 
pions, nucleons and $\Delta$ and Roper resonances relevant for the presented calculations as well as all notations and details of calculations can be found in Refs. [2-4].

\section{$2 \pi N$ scattering}

The amplitude of $\pi^{a}(q)+N(p) \rightarrow \pi^{a^{\prime}}\left(q^{\prime}\right)+N\left(p^{\prime}\right)$ scattering in the isospin limit can be parameterized as:

$$
T_{\pi N}^{a^{\prime} a}(s, t, u)=\chi_{N^{\prime}}^{\dagger}\left\{\delta_{a^{\prime} a} T^{+}(s, t, u)+\frac{1}{2}\left[\tau_{a^{\prime}}, \tau_{a}\right] T^{-}(s, t, u)\right\} \chi_{N},
$$

where $a^{\prime}$ and $a$ are Cartesian isospin indices, $\tau_{i}$ - Pauli matrices and $\chi_{N}, \chi_{N^{\prime}}$ denote nucleon iso-spinors. Lorentz decomposition of $T^{ \pm}$, well suited for chiral EFT, has the form:

$$
T^{ \pm}(s, t, u)=\bar{u}^{\left(s^{\prime}\right)}\left(p^{\prime}\right)\left\{D^{ \pm}(s, t, u)-\frac{1}{4 m_{N}}\left[\phi^{\prime}, \phi\right] B^{ \pm}(s, t, u)\right\} u^{(s)}(p),
$$

with $\left(s^{\prime}\right),(s)$ denoting the spins of $\bar{u}, u$, respectively.

For diagrams involving only pion and nucleon lines, we use the standard power counting of Refs. [5, 6] considering the pion mass $M_{\pi}$ and small momenta as of order $O(p)$. For diagrams with delta lines we apply the power counting of Ref. [7], that is we count $m_{\Delta}-m_{N}$ as of order $O(p)$. For $s \rightarrow m_{\Delta}^{2}$ delta-propagator diverges, therefore we need to sum up self-energy insertions, i.e. consider the dressed propagator $D^{\mu \nu}(k) \sim 1 /\left(k-m_{\Delta}-\Sigma(k)\right) \sim 1 /(-\Sigma(k)) \sim 1 / p^{3}$, unless, as we did in our work, one uses the complex-mass scheme where the undressed propagator contains the width of the unstable particle and therefore the re-summation is not necessary.

To renormalize the loop diagrams we applied EOMS renormalization scheme of Refs. [8, 9] and its generalization for delta [10].
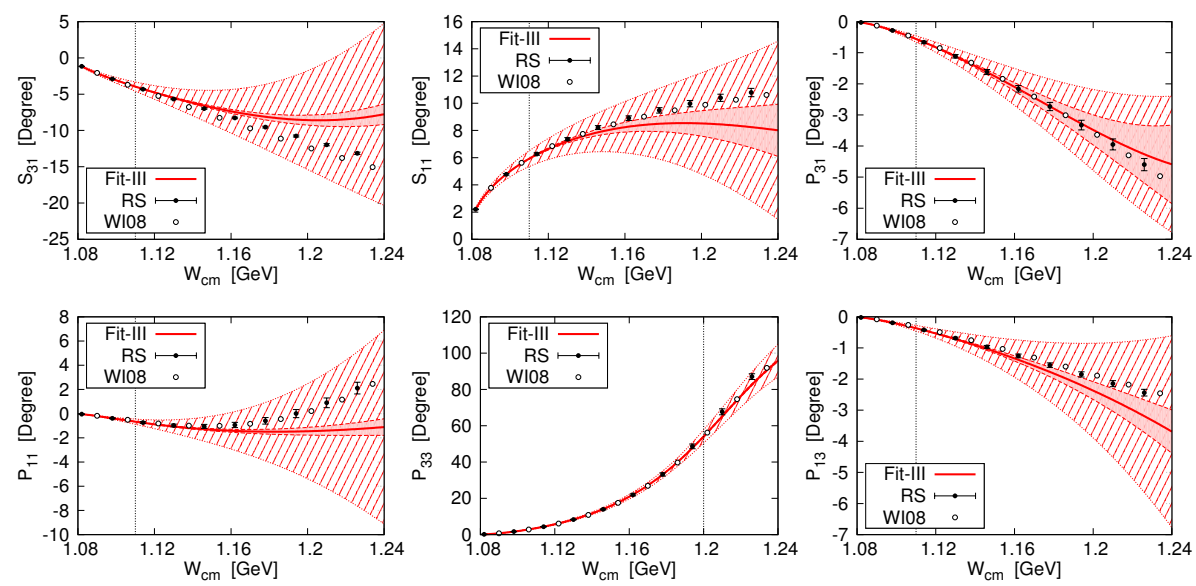

Figure 1. Phase shifts corresponding to Fit-III. Dots with error bars represent the RS phase shifts; Circles - GWU phase shifts. The red line is the result of Fit III. The red narrow error bands are uncertainties propagated from the errors of LECs. The wide dashed error bands are theoretical uncertainties.

We fitted the unknown low-energy constants (LECs) to the phase shifts of the $S$ and $P$ partial waves. Then we predicted the $D$ - and $F$-wave phase shifts using the determined 
LECs. Unfortunately, none of the available partial wave (PW) analysis of the $\pi N$ amplitudes provide uncertainties. Therefore, we performed fits to the phase shifts generated by the recent RS-equation analysis of the $\pi N$ scattering [11]. There are eleven LECs involved in our $\pi N$ amplitudes in total: $c_{1}, c_{2}, c_{3}, c_{4}, d_{1}+d_{2}, d_{3}, d_{5}, d_{14}-d_{15}, g_{\pi N}, g_{\pi N \Delta}$ and $g_{1}$. We fixed $g_{\pi N}$ coupling to the value $g_{\pi N}^{2} /(4 \pi)=13.69 \pm 0.20[12,13]$. We carried out three fits. Fit-I was done for the delta-less case. In Fit II the leading order tree contributions of the delta-exchange diagrams were added to the delta-less case and it served only the purpose of estimating the effect of the loop diagrams with delta. Fit-III was done with the full contributions of pions, nucleons and deltas up to next-to-next-to-leading-order. All the obtained $c_{i}$ and most of the higher order LECs are of natural size. Our plots for Fit-III are shown in Fig. 1.

Using the fitted LECs we calculated the phase shifts of higher PWs shown in Fig. 2. Except for $D_{33}$ channel, our predictions agree qualitatively with the GWU results [17] and the predictions of the delta-full theory are slightly better than those of the delta-less theory.
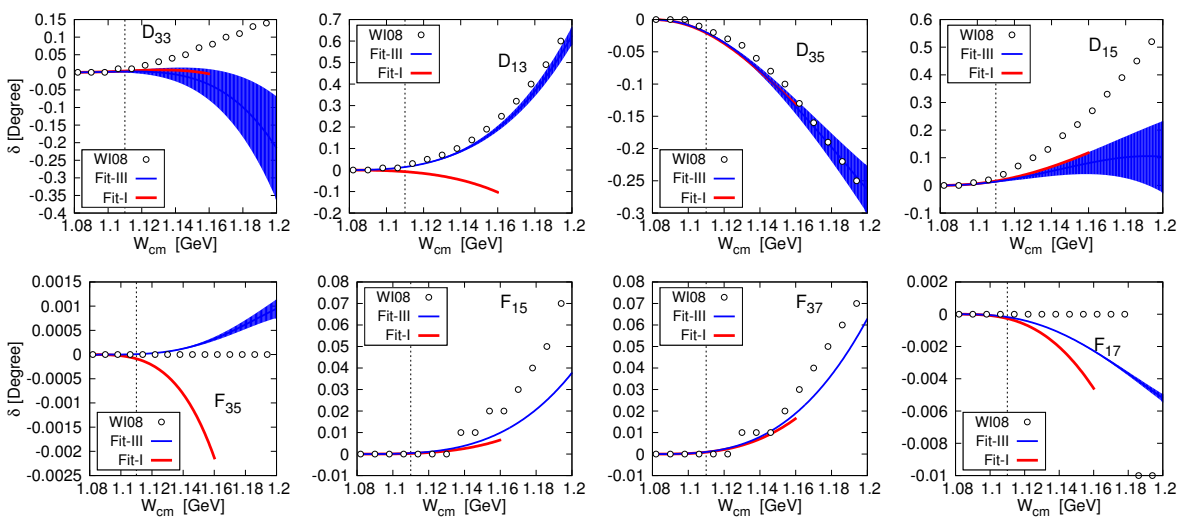

Figure 2. Phase shifts of the $D$ and $F$ PWs from the delta-less and delta-full BChPT corresponding to Fit-I (red) and Fit-III (blue), respectively. The circles correspond to phase shifts by the GWU group.

\section{The width of the Roper resonance}

In this section we briefly present the result of order $\delta^{5}$ calculation of the width of the Roper resonance which corresponds to leading two-loop order in BChPT of pions, nucleons and the delta and Roper resonances [4]. Here we consider $m_{R}-m_{N} \sim 400 \mathrm{MeV}$ as a small parameter of the order $\delta^{1}$ and count $M_{\pi} \sim \delta^{2}$. We also count $m_{R}-m_{\Delta} \sim m_{\Delta}-m_{N} \sim \delta^{2}$.

The physical mass and the width of the Roper resonance are defined by parameterizing the pole of the propagator as

$$
z=m_{R}-i \frac{\Gamma_{R}}{2} .
$$

The pole of the dressed propagator of the Roper resonance $S_{R}$ is obtained by solving

$$
S_{R}^{-1}(z) \equiv z-m_{R 0}-\Sigma_{R}(z)=0
$$

where $-i \Sigma_{R}(\not p)$ is the self-energy. We parameterize the pole as

$$
z=m_{2}+\hbar \delta z_{1}+\hbar^{2} \delta z_{2}+O\left(\hbar^{3}\right)
$$


where $m_{2}=m_{R}^{0}+4 c_{1}^{R} M^{2}$, with $m_{R}^{0}$ the Roper mass in the chiral limit and write the self-energy as an expansion:

$$
\Sigma_{R}=\hbar \Sigma_{1}+\hbar^{2} \Sigma_{2}+O\left(\hbar^{3}\right) .
$$

By expanding Eq.(1) in powers of $\hbar$, we get

$$
\hbar \delta z_{1}+\hbar^{2} \delta z_{2}-\hbar \Sigma_{1}\left(m_{2}\right)-\hbar^{2} \delta z_{1} \Sigma_{1}^{\prime}\left(m_{2}\right)-\hbar^{2} \Sigma_{2}\left(m_{2}\right)+O\left(\hbar^{3}\right)=0 .
$$

Solving Eq. (2) order by order we obtain

$$
\begin{aligned}
& \delta z_{1}=\Sigma_{1}\left(m_{2}\right), \\
& \delta z_{2}=\Sigma_{1}\left(m_{2}\right) \Sigma_{1}^{\prime}\left(m_{2}\right)+\Sigma_{2}\left(m_{2}\right) .
\end{aligned}
$$

The width takes the form

$$
\begin{aligned}
\Gamma_{R} & =\hbar 2 i \operatorname{Im}\left[\Sigma_{1}\left(m_{2}\right)\right] \\
& +\hbar^{2} 2 i\left\{\operatorname{Im}\left[\Sigma_{1}\left(m_{2}\right)\right] \operatorname{Re}\left[\Sigma_{1}^{\prime}\left(m_{2}\right)\right]+\operatorname{Re}\left[\Sigma_{1}\left(m_{2}\right)\right] \operatorname{Im}\left[\Sigma_{1}^{\prime}\left(m_{2}\right)\right]\right\} \\
& +\hbar^{2} 2 i \operatorname{Im}\left[\Sigma_{2}\left(m_{2}\right)\right]+O\left(\hbar^{3}\right) .
\end{aligned}
$$

The contribution of the second term in Eq. (3) is beyond the accuracy of our calculation. To calculate the contributions of the first and third terms we use the Cutkosky cutting rules. Only contributions obtained by cutting the lines, corresponding to stable particles, are needed.

The contributions of the one- and two-loop self-energy diagrams in the width of the Roper resonance at order $\delta^{5}$ sum up to

$$
\Gamma_{R}=\Gamma_{R \rightarrow \pi N}+\Gamma_{R \rightarrow \pi \pi N}
$$

The $R(p) \rightarrow N\left(p^{\prime}\right) \pi^{a}(q)$ decay width reads

$$
\Gamma_{R \rightarrow \pi N}=\frac{\lambda^{1 / 2}\left(m_{R}^{2}, m_{N}^{2}, M^{2}\right)}{16 \pi m_{R}^{3}}\left|\mathcal{M}_{1}\right|^{2}
$$

with $\lambda(x, y, z)=(x-y-z)^{2}-4 y z$ and $\mathcal{M}_{1}$ the corresponding decay amplitude.

The decay width corresponding to the $\pi \pi N$ final state is given by:

$$
\Gamma_{R \rightarrow \pi \pi N}=\frac{1}{32 m_{R}^{3}(2 \pi)^{3}} \int_{4 M_{\pi}^{2}}^{\left(m_{R}-m_{N}\right)^{2}} \mathrm{~d} s_{1} \int_{s_{2-}}^{s_{2+}} \mathrm{d} s_{2}|\mathcal{M}|^{2},
$$

where $\mathcal{M}$ is the $R(p) \rightarrow N\left(p^{\prime}\right) \pi^{a}\left(q_{1}\right) \pi^{b}\left(q_{2}\right)$ decay amplitude and

$$
s_{2 \pm}=\frac{m_{R}^{2}+m_{N}^{2}+2 M_{\pi}^{2}-s_{1}}{2} \pm \frac{1}{2 s_{1}} \lambda^{1 / 2}\left(s_{1}, m_{R}^{2}, m_{N}^{2}\right) \lambda^{1 / 2}\left(s_{1}, M_{\pi}^{2}, M_{\pi}^{2}\right) .
$$

The delta propagators in leading order tree diagrams contributing to the $R \rightarrow \pi \pi N$ decay are dressed and loop diagrams do not contribute at order $\delta^{5}$.

For numerical results we used the following values of the parameters from PDG [18]

$$
\begin{gathered}
M_{\pi}=139 \mathrm{MeV}, m_{N}=939 \mathrm{MeV}, m_{\Delta}=1210 \pm 1 \mathrm{MeV} \\
\Gamma_{\Delta}=100 \pm 2 \mathrm{MeV}, m_{R}=1365 \pm 15 \mathrm{MeV}, F_{\pi}=92.2 \mathrm{MeV}
\end{gathered}
$$

Further, we substituted $g_{A}=1.27$ and $h=1.42 \pm 0.02$ from Ref.[2]. We fixed $g_{\pi N R}$ by reproducing $\Gamma_{R \rightarrow \pi N}=(123.5 \pm 19.0) \mathrm{MeV}$ from PDG, obtaining $g_{\pi N R}= \pm(0.47 \pm 0.11)$. Next, following Ref. [14] we assumed $g_{R}=g_{A}$ and $h_{R}=h$ and obtained:

$$
\begin{aligned}
\Gamma_{R \rightarrow \pi \pi N} & =[0.53(32)-0.98(60)+0.53(32) \pm 3.57(1.41) \\
& \mp 4.57(1.97)+40.4(22.2)] \mathrm{MeV}=40.5(22.3) \mathrm{MeV}
\end{aligned}
$$


The largest contribution in Eq. (4) comes from the decay diagram with intermediate $\Delta$ state. Further, using the approach of Ref. [15] we estimated the theoretical error due to the omitting the higher order contributions and obtained

$$
\Gamma_{R \rightarrow \pi \pi N}=(40.5 \pm 22.3 \pm 16.8) \mathrm{MeV}
$$

which is consistent with $\Gamma_{\pi \pi N}=(66.5 \pm 9.5) \mathrm{MeV}$ quoted by PDG [18].

\section{Width of the $\Delta$-resonance}

Analogously to the Roper resonance the pole mass and the width of delta are defined by parameterizing the pole of the propagator as [19]

$$
z=m_{\Delta}-i \frac{\Gamma_{\Delta}}{2} .
$$

The pole position of the $\Delta$-propagator can be found by solving equation

$$
z-m_{\Delta}^{0}-\Sigma_{1}\left(z^{2}\right)-z \Sigma_{6}\left(z^{2}\right) \equiv z-m_{\Delta}^{0}-\Sigma(z)=0,
$$

where the self-energy of the $\Delta$-resonance is parameterised as:

$$
\begin{aligned}
\Sigma^{\mu \nu} & =\Sigma_{1}\left(p^{2}\right) g^{\mu \nu}+\Sigma_{2}\left(p^{2}\right) \gamma^{\mu} \gamma^{v}+\Sigma_{3}\left(p^{2}\right) p^{\mu} \gamma^{v} \\
& +\Sigma_{4}\left(p^{2}\right) \gamma^{\mu} p^{v}+\Sigma_{5}\left(p^{2}\right) p^{\mu} p^{v}+\Sigma_{6}\left(p^{2}\right) \not p g^{\mu \nu}+\Sigma_{7}\left(p^{2}\right) \not p \gamma^{\mu} \gamma^{v} \\
& +\Sigma_{8}\left(p^{2}\right) \not p p^{\mu} \gamma^{v}+\Sigma_{9}\left(p^{2}\right) \not p \gamma^{\mu} p^{v}+\Sigma_{10}\left(p^{2}\right) \not p p^{\mu} p^{v} .
\end{aligned}
$$

We calculated $z$ perturbatively order by order in loop expansion (i.e. expansion in powers of $\hbar)$. For that purpose we wrote

$$
\Sigma=\hbar \Sigma_{(1)}+\hbar^{2} \Sigma_{(2)}+O\left(\hbar^{3}\right)
$$

and substituting in Eq. (5) obtained the following expression for the width

$$
\begin{aligned}
\Gamma_{\Delta} & =\hbar 2 i \operatorname{Im}\left[\Sigma_{(1)}\left(m_{\Delta}\right)\right]+\hbar^{2} 2 i\left\{\operatorname{Im}\left[\Sigma_{(1)}\left(m_{\Delta}\right)\right] \operatorname{Re}\left[\Sigma_{(1)}^{\prime}\left(m_{\Delta}\right)\right]\right. \\
& \left.+\operatorname{Re}\left[\Sigma_{(1)}\left(m_{\Delta}\right)\right] \operatorname{Im}\left[\Sigma_{(1)}^{\prime}\left(m_{\Delta}\right)\right]\right\}+\hbar^{2} 2 i \operatorname{Im}\left[\Sigma_{(2)}\left(m_{\Delta}\right)\right]+O\left(\hbar^{3}\right) .
\end{aligned}
$$

For $\Sigma_{(1)}$ we used the corresponding explicit expressions. For leading two-loop contribution to the width we used the Cutkosky cutting rules, that is we related it to $A_{\Delta \rightarrow \pi N}$ amplitude via

$$
\Gamma_{\Delta}=\frac{\left[\left(m_{\Delta}+m_{N}\right)^{2}-M_{\pi}^{2}\right]\left[\left(m_{\Delta}^{2}-m_{N}^{2}-M_{\pi}^{2}\right)^{2}-4 M_{\pi}^{2} m_{N}^{2}\right]^{3 / 2}}{192 \pi m_{\Delta}^{5}}\left|A_{\Delta \rightarrow \pi N}\right|^{2} .
$$

Calculating one- and two-loop contributions in the delta width we obtained:

$$
\Gamma_{\Delta}=53.91 h_{A}^{2}+0.87 g_{1}^{2} h_{A}^{2}-3.31 g_{1} h_{A}^{2}-0.99 h_{A}^{4} .
$$

Substituting $\Gamma_{\Delta}=100 \pm 2 \mathrm{MeV}$ from the PDG we extracted $h_{A}$ as a function of the leading order $\pi \Delta$ coupling constant $g_{1}$. The obtained result is plotted in Fig. 3.

This work was supported in part by Georgian Shota Rustaveli National Science Foundation (Grant No. FR17-354) and by the DFG (TR 16 and CRC 110). The work of UGM was also supported by the Chinese Academy of Sciences (CAS) President's International Fellowship Initiative (PIFI) (Grant No. 2015VMA076). 


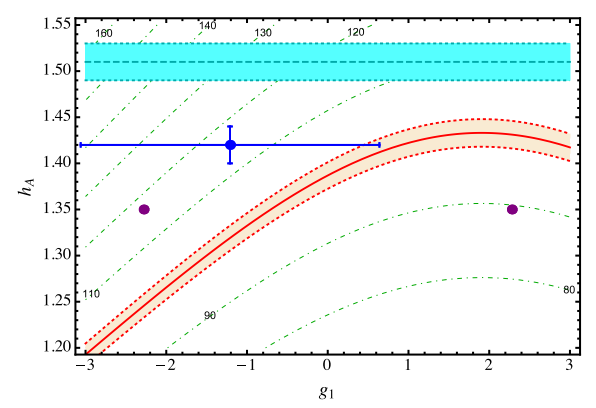

Figure 3. $h_{A}$ as a function of $g_{1}$. The central line corresponds to $\Gamma_{\Delta}=100 \mathrm{MeV}$, while the band is obtained by varying $\Gamma_{\Delta} \sim 98-102 \mathrm{MeV}$. The dot-dashed lines correspond to delta widths indicated by their values. The blue dot with error bars represents the real part of the coupling fitted to $\pi N$ scattering, the purple dots stand for the leading order $\pi N \Delta$ coupling in the large- $N_{c}$ limit and the horizontal dashed line with cyan band corresponds to the value (with error represented by the band) from Ref. [16]

\section{References}

[1] S. Weinberg, Physica A 96, 327 (1979).

[2] D. L. Yao, D. Siemens, V. Bernard, E. Epelbaum, A. M. Gasparyan, J. Gegelia, H. Krebs and U.-G. Meißner, JHEP 1605, 038 (2016).

[3] J.Gegelia, U.-G.Meißner, D. Siemens, D.L. Yao, Phys. Lett. B 763, 1 (2016).

[4] J. Gegelia, U.-G. Meißner and D. L. Yao, Phys. Lett. B 760, 736 (2016).

[5] S. Weinberg, Nucl. Phys. B 363, 3 (1991).

[6] G. Ecker, Prog. Part. Nucl. Phys. 35, 1 (1995).

[7] T. R. Hemmert, B. R. Holstein and J. Kambor, J. Phys. G 24, 1831 (1998).

[8] J. Gegelia and G. Japaridze, Phys. Rev. D 60, 114038 (1999).

[9] T. Fuchs, J. Gegelia, G. Japaridze and S. Scherer, Phys. Rev. D 68, 056005 (2003).

[10] C. Hacker, N. Wies, J. Gegelia and S. Scherer, Phys. Rev. C 72, 055203 (2005).

[11] M. Hoferichter, J. Ruiz de Elvira, B. Kubis and U.-G. Meißner, Phys. Rept. 625, 1 (2016).

[12] V. Baru, C. Hanhart, M. Hoferichter, B. Kubis, A. Nogga and D. R. Phillips, Phys. Lett. B 694, 473 (2011).

[13] V. Baru, C. Hanhart, M. Hoferichter, B. Kubis, A. Nogga and D. R. Phillips, Nucl. Phys. A 872, 69 (2011).

[14] S. R. Beane and U. van Kolck, J. Phys. G 31, 921 (2005).

[15] E. Epelbaum, H. Krebs and U.-G. Meißner, Eur. Phys. J. A 51, no. 5, 53 (2015).

[16] V. Bernard, E. Epelbaum, H. Krebs and U.-G. Meißner, Phys. Rev. D 87, no. 5, 054032 (2013).

[17] R. A. Arndt, W. J. Briscoe, I. I. Strakovsky and R. L. Workman, Phys. Rev. C 74, 045205 (2006).

[18] C. Patrignani et al. [Particle Data Group], Chin. Phys. C 40, no. 10, 100001 (2016).

[19] D. Djukanovic, J. Gegelia and S. Scherer, Phys. Rev. D 76, 037501 (2007). 